\title{
Experimental modelling of a mine working failure under nonuniformly distributed compression
}

\author{
Sergey Suknev ${ }^{1^{*}}$ \\ ${ }^{1}$ Chersky Institute of Mining of the North, Siberian Branch, Russian Academy of Sciences, 677980 \\ Yakutsk, Russia
}

\begin{abstract}
This paper presents a theoretical and experimental study of the fracture of a quasi-brittle geomedium containing a cylindrical opening and subjected to a nonuniformly distributed compressive load. Laboratory tests are carried out using hydrated gypsum plaster as a model rock-like material. High-strength plaster specimens demonstrate brittle fracture, while specimens made of plaster of Paris show quasi-brittle fracture. In this case, the application of the known criteria does not allow obtaining satisfactory estimates of the fracture load. New nonlocal fracture criteria are proposed, which are the development of the average stress criterion, and point stress criterion, and which contain a complex parameter that characterizes the size of the fracture process zone and accounts not only for the material microstructure, but also plastic properties of the material, geometry of the opening, and loading conditions. The calculation results are in good agreement with the resulting experimental data.
\end{abstract}

\section{Introduction}

The tensile strength of rocks is much lower than their compressive strength. At the same time, tensile stress zones and tensile cracks (gaps) may be formed near mine workings even under compressive loads, which is very dangerous. Moreover, similarly to structurally heterogeneous materials, the strength properties of rocks depend on a loaded volume (size effect), which is manifested the most under stress concentrations where the effective loaded volume is determined by the stress concentration zone whose size is small as compared to the dimensions of a deformed solid block of rock.

The effect of boundary conditions on the fracture of brittle geomaterial in the stress concentration zone under a biaxial load with allowance for the size effect was studied in [1]. It was shown on the basis of those experimental and theoretical studies that the influence of loading conditions on the critical (failure) stress could be well-described by nonlocal fracture criteria, developed in [2-14] and other works. Nonlocal criteria are based on the concept of the formation of a fracture process zone in the material, in which there is local redistribution of stresses, whereas the main material is deformed elastically until fracture. A common property of these criteria is the introduction of intrinsic material length

\footnotetext{
*Corresponding author: suknyov@igds.ysn.ru
} 
$d_{0}$ that characterizes its microstructure. As noted in [15], the field of application of nonlocal criteria is predominantly the brittle fracture of materials.

This paper presents the theoretical and experimental study of the influence of the diameter of a circular hole that simulates mine working on the fracture of a rock-like material in the stress concentration zone under nonuniformly distributed compression and the analysis of the application of nonlocal criteria for describing the fracture of a quasibrittle geomedium containing a cylindrical opening.

\section{Experimental technique and materials}

Experimental studies include two series of tests of plaster specimens with circular holes under nonuniformly distributed compression. The specimen is subjected to load $p$ via rigid inserts placed between the specimen and compression platens (Fig. 1).

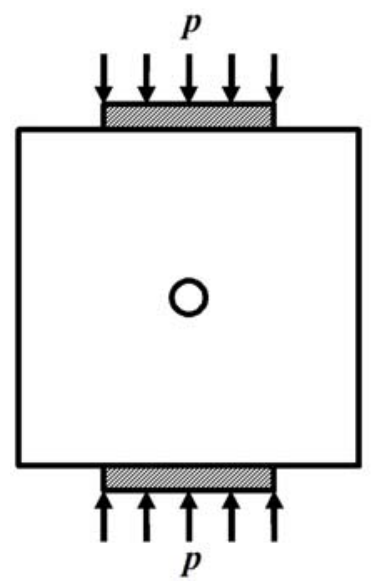

Fig. 1. Scheme of the specimen loading.

Tests are carried out using specimens made of high-strength gypsum plaster (plaster 1) for the first series of experiments and conventional plaster of Paris (plaster 2) for the second series of experiments. The specimens are 200x200-mm square plates with thicknesses of $40 \mathrm{~mm}$ (plaster 1) and $36 \mathrm{~mm}$ (plaster 2). Before the beginning of the tests, a circular hole with a diameter ranging from 1 to $20 \mathrm{~mm}$ is drilled at the center of each specimen. The specimens are loaded via the inserts $120-\mathrm{mm}$ in size. During the tests, the sudden initiation of tensile cracks in the tensile stress concentration zones on the hole boundary is observed. Crack initiation is also accompanied by the local unloading of the specimen, which is reflected by the appearance of a "tooth" (sharp drop) on the stress-strain diagram. The critical load at the instance of crack initiation is determined by the peak of the "tooth" on the diagram.

The compressive strength of the material is also determined using 200x200-mm square specimens with no holes. They are loaded via $200-\mathrm{mm}$ inserts. The uniaxial compressive strength is $34.11 \mathrm{MPa}$ for plaster 1 and $11.53 \mathrm{MPa}$ for plaster 2 . The tensile strength of the material is determined by subjecting dog-bone shaped specimens to tensile loads. The uniaxial tensile strength is $5.38 \mathrm{MPa}$ for plaster 1 and 2.61 MPa for plaster 2. 


\section{Theoretical approach}

The critical pressure is calculated using conventional and modified average stress criteria and point stress criteria [15].

It is shown in [1] that loading the specimen according to the scheme illustrated in Fig. 1 causes a uniform biaxial stress state at the central part (outside the influence of the hole): tension with stresses $\alpha \sigma$ along the horizontal axis of the specimen and compression with stresses $\sigma$ along its vertical axis. The values of $\sigma$ and $\alpha$ are calculated by the finite element method at the center of the specimen loaded via the inserts of a given size and having no hole. For the inserts used in the experiments, the value of $\sigma$ is $0.764 p$ and $\alpha=0.187$.

The average stress criterion (ASC) has the form $\left\langle\sigma_{e}\right\rangle_{d}<\sigma_{0}$, where $\left\langle\sigma_{e}\right\rangle_{d}$ is the value of the equivalent stress, which is averaged at distance $d$ along the dangerous section, and $\sigma_{0}$ is the tensile strength of the material. For brittle materials, $d=d_{0}=$ const. The equivalent stress is determined from the theory of maximum tensile stress. The critical stress at which tensile cracks are initiated from the hole is determined by the expression [1]

$$
\sigma_{c}=2 \sigma_{0}\left[(1+\gamma) \gamma^{-3}+\alpha\left(1+\gamma^{-1}\right)\left(2+\gamma^{-2}\right)\right]^{-1},
$$

where $\gamma=1+d / a$, and $a$ is the radius of the hole. If $\gamma=1$, Eq. (1) yields the calculation of the critical stress according to a traditional (local) fracture criterion.

In order to describe a quasi-brittle fracture, the averaging size is determined from the equation [15]

$$
d=d_{0}+\beta L_{e}
$$

where $L_{e}=\frac{\sigma_{e}}{\left|\operatorname{grad} \sigma_{e}\right|}$ is the size of the stress concentration zone, and $\beta$ is the dimensionless parameter characterizing the plasticity of the material. For brittle materials, $\beta=0$; for ductile materials, $\beta>>1$. If $\beta \sim 1$, the material is characterized by moderate plastic properties.

The size of the stress concentration zone, calculated with allowance for the known solution of the Kirsch problem [16], is $L_{e}=a \frac{1+3 \alpha}{5+7 \alpha}$. Accordingly, the expression for $\gamma$ takes the form:

$$
\gamma=1+\frac{d_{0}}{a}+\beta \frac{1+3 \alpha}{5+7 \alpha} .
$$

In accordance with Eq. (1) and with account for the estimates for $\sigma$ and $\alpha$, the expression for the critical pressure in the specimen with a circular hole is written as

$$
p_{c}=2 \chi C_{0}\left[0.764(1+\gamma) \gamma^{-3}+0.143\left(1+\gamma^{-1}\right)\left(2+\gamma^{-2}\right)\right]^{-1},
$$

where $\chi=\sigma_{0} / C_{0}$, and $C_{0}$ is the compressive strength of the material. The parameter $\gamma$ is determined by Eq. (3), in which $\alpha=0.187$. For quasi-brittle materials known for moderate 
plastic properties, the asymptotic (as $a \rightarrow \infty$ ) value of the critical pressure is approximately $T_{s} \approx T_{0}(1+\beta / 2)$, where $T_{0}=0.838 \chi C_{0}$.

Integration in the point stress criterion (PSC) is replaced by the calculation of the equivalent stress $\sigma_{e}$ at some point located at the distance $d$ from the maximum point. The strength criterion takes the form $\sigma_{e}\left(x_{0}+d\right)<\sigma_{0}$, where $x_{0}$ is the coordinate of the maximum point of the equivalent stress. The parameter $d$ is also assumed to be the constant of the material, which does not actually coincide with a similar parameter in the ASC. The critical stress for the specimen with a circular hole, subjected to nonuniformly distributed compression, is determined from the expression (Fig. 1) [1]:

$$
\sigma_{c}=2 \sigma_{0}\left[\left(-\gamma^{-2}+3 \gamma^{-4}\right)+\alpha\left(2+\gamma^{-2}+3 \gamma^{-4}\right)\right]^{-1} .
$$

For quasi-brittle materials, $\gamma$ is calculated from Eq. (3).

In accordance with Eq. (5) and with account for the estimates for $\sigma$ and $\alpha$, the expression for the critical pressure in the specimen with a circular hole on the basis of the PSC is written as follows:

$$
p_{c}=2 \chi C_{0}\left[0.764\left(-\gamma^{-2}+3 \gamma^{-4}\right)+0.143\left(2+\gamma^{-2}+3 \gamma^{-4}\right)\right]^{-1} .
$$

For quasi-brittle materials, characterized by moderate plastic properties, $T_{s} \approx T_{0}(1+\beta)$.

\section{Results}

Figure 2 illustrates experimental data (points) on the value of the load at the instance of tensile crack initiation from the hole as a function of the hole diameter $l$, obtained on the specimens made of plaster 1 , and the results of the calculation of the critical pressure (curve) according to the ASC on the basis of Eq. (4) for $\beta=0$. The size $d_{0}$ is $0.6 \mathrm{~mm}$. The dashed curve is calculated according to the traditional approach. Figure 3 shows experimental data (points) and the results of the calculation of the critical pressure for plaster 2 for $\beta=0$ (curve 1 ) and $\beta=0.6$ (curve 2). The size $d_{0}$ is $1.0 \mathrm{~mm}$. The stress $T_{s}$ is equal to $T_{0}$ in the first case (dashed curve) and $T_{s}=1.3 T_{0}$ in the second case (solid curve).

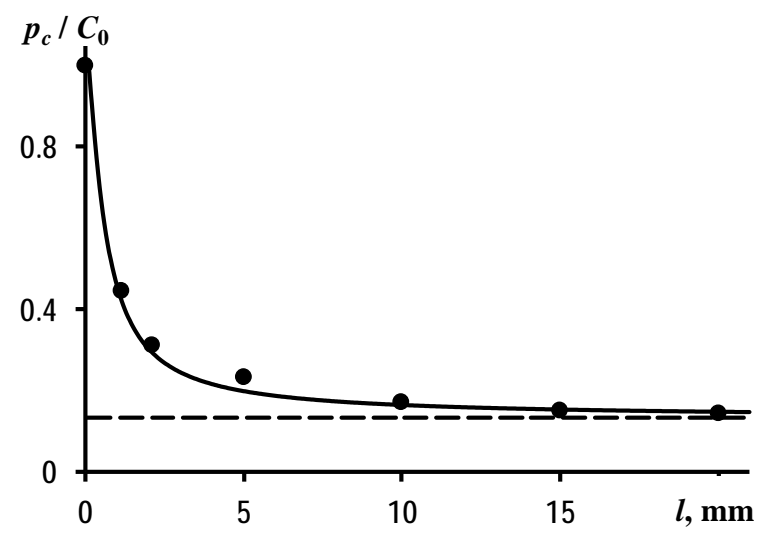

Fig. 2. Critical pressure versus the hole diameter for the specimens of plaster 1 . The calculations are based on the ASC. 


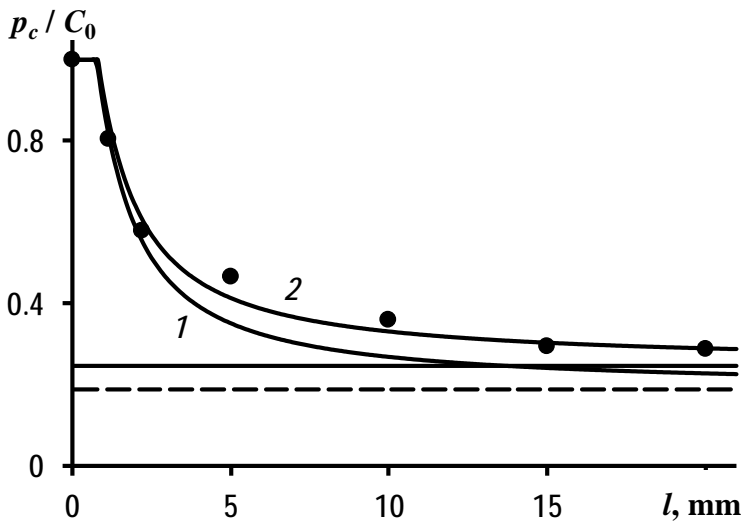

Fig. 3. Critical pressure versus the hole diameter for the specimens of plaster 2 . The calculations are based on the ASC.

Figure 4 shows the experimental data (points) referring to the value of the load at the instance of tensile crack initiation from the hole as a function of the hole diameter $l$, obtained using the specimens of plaster 1 , and the results of the calculation of the critical pressure (curve) according to the PSC on the basis of Eq. (6) for $\beta=0$. The size $d_{0}$ is $0.25 \mathrm{~mm}$. The dashed curve is calculated according to the traditional approach. Figure 5 shows the experimental data (points) and the results of the calculation of the critical pressure for plaster 2 for $\beta=0$ (curve 1 ) and $\beta=0.3$ (curve 2). The size $d_{0}$ is $0.3 \mathrm{~mm}$. The stress $T_{s}$ is equal to $T_{0}$ in the first case (dashed curve) and $T_{s}=1.3 T_{0}$ in the second case (solid curve).

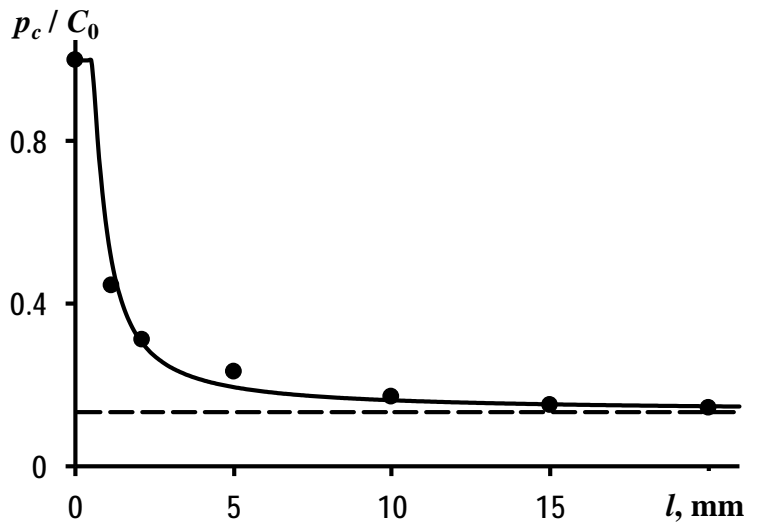

Fig. 4. Critical pressure versus the hole diameter for the specimens of plaster 1 . The calculations are based on the PSC. 


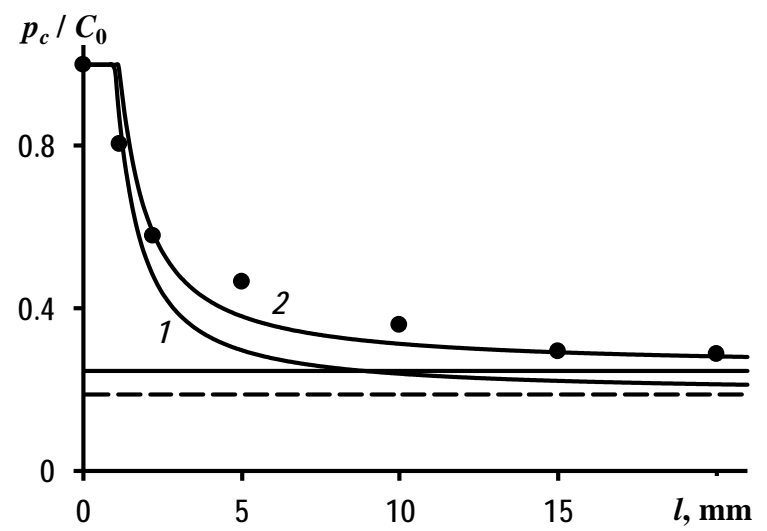

Fig. 5. Critical pressure versus the hole diameter for the specimens of plaster 2 . The calculations are based on the PSC.

\section{Discussion}

Figures 2-5 illustrate a significant size effect, i.e., the influence of the hole diameter on the local strength of the material. As it becomes smaller, the critical pressure rises, reaching the compressive strength. As it becomes larger, the critical pressure asymptotically tends to the stress $T_{0}$ for plaster 1 and stress $T_{s}$ for plaster 2 . The fracture of the specimens made of plaster 1 , characterized by the sudden initiation and rapid propagation of tensile cracks from the hole along the compression axis, can be described within the framework of known nonlocal criteria. The experimental data confirm the fact that the critical pressure asymptotically tends to the value calculated in accordance with the traditional approach for an elastic solid. All this allows characterizing the fracture of this material as brittle.

At the same time, the application of criteria for describing experimental data obtained using the specimens of plaster 2 results in satisfactory estimates of the value of the critical pressure only for small (1-2 $\mathrm{mm}$ ) diameters of the hole. The calculation results obtained for large diameters of the hole show low values of the critical pressure. The resulting experimental data indicate that, as the hole diameter increases, the critical pressure asymptotically tends to a value exceeding the value determined for an elastic solid by $30 \%$. However, as in the first case, the fracture of the specimens of plaster 2 is characterized by the sudden initiation of tensile cracks that rapidly propagate along the compression axis. All this allows characterizing the fracture of this material in the studied range of the hole diameters as quasi-brittle. This behavior of the critical pressure is well-described by the modified nonlocal criteria in which the size of the fracture process zone $d$ is determined from Eq. (2).

\section{Conclusion}

The fracture of the rock-like materials with a circular hole under nonuniformly distributed compression is theoretically and experimentally studied, and the possibility of applying nonlocal criteria to estimate the fracture load is analyzed. It is determined in the laboratory tests that the specimens made of high-strength plaster demonstrate brittle fracture, in which case the critical pressure can be calculated within the framework of the known nonlocal fracture criteria. The specimens made of the plaster of Paris show quasi-brittle fracture. In this case, the application of the known criteria does not allow obtaining satisfactory 
estimates of the fracture load. Therefore, it is proposed to calculate the fracture load using the modified nonlocal fracture criteria, which are the development of the average stress criterion and point stress criterion, and which contain a complex parameter that characterizes the size of the fracture process zone and accounts for the microstructure and plastic properties of the material, specimen geometry, and its loading conditions. The calculation results based on the modified criteria are in good agreement with the resulting experimental data.

The research was supported by the Russian Foundation for Basic Research under grant number 18-0500323.

\section{References}

1. S.V. Suknev, J. Appl. Mech. Tech. Phys. 56, 6, 1078 (2015)

2. V.V. Novozhilov, J. Appl. Math. Mech. 33, 201 (1969)

3. J.M. Whitney, R.J. Nuismer, J. Compos. Mater. 8, 253 (1974)

4. S.K. Maiti, R.A. Smith, Int. J. Fract. 23, 4, 281 (1983)

5. A. Seweryn, Z. Mroz, Eng. Fract. Mech. 51, 6, 955 (1995)

6. S.E. Mikhailov, Eng. Fract. Mech. 52, 4, 731 (1995)

7. A.V. Dyskin, Int. J. Fract. 83, 2, 191 (1997)

8. J. Toribio, Eng. Fract. Mech. 57, 4, 391 (1997)

9. D. Leguillon, Eur. J. Mech. A/Solids 21, 1, 61 (2002)

10. D. Taylor, P. Cornetti, N. Pugno, Eng. Fract. Mech. 72, 7, 1021 (2005)

11. D. Taylor, The theory of critical distances: a new perspective in fracture mechanics (Elsevier, Oxford, 2007)

12. B. Lecampion, Int. J. Rock Mech. Min. Sci. 56, 67 (2012)

13. A.R. Torabi, E. Pirhadi, Eur. J. Mech. A/Solids 49, 1 (2015)

14. A. Sapora, A.R. Torabi, S. Etesam, P. Cornetti, Fatigue Fract. Eng. Mater. Struct. 41, 7, 1627 (2018)

15. S.V. Suknev, Phys. Mesomech. 22, 6, 504 (2019)

16. S.P. Timoshenko, J.N. Goodier, Theory of elasticity (McGraw-Hill, New York, 1970) 